\title{
Summing up
}

\author{
T. KEITH LYLE \\ London
}

The aim of this meeting was to bring together some of those currently engaged in laboratory research into the problems of strabismus and clinicians concerned with its treatment so that each group could learn something of what the others are doing. Conclusions reached in the laboratory with regard to visual function may be of the greatest help in understanding clinical problems and in planning practical methods to restore normal function.

Dr. von Noorden has demonstrated, for the first time, that in experimental uniocular amblyopia in the baboon, achieved by uniocular tarsorrhaphy, there occurred a definite pathological shrinkage of cells in the lateral geniculate nucleus-a change which was reversible after enforcing the use of the amblyopic eye by occlusion of the normal eye. This pathological change did not, however, occur if the eyelids of both eyes were subjected to tarsorrhaphy.

Dr. Ikeda's work in the cat showed that there was a difference in function between the cells in the central area of the retina and those in the peripheral retina. The former show sustained "firing" of a tonic and slow type and are concerned with spatial discrimination, whereas the latter show a slow and intermittent firing which is phasic and fast and concerned more with localization and with the act of fixation and re-fixation. She showed that, in the cat, the central cells were adversely affected in their action if images presented to them were made blurred by a "defocusing" mechanism (using strong plus spherical lenses), thus demonstrating the importance of the correction of refractive errors in strabismic, and especially in anisometropic, amblyopia.

Dr. Blakemore showed that in binocular animals both visual cortices are "driven" by both eyes, those on the temporal periphery being "driven" by the contralateral eye and those on the nasal periphery by the ipsilateral eye, while the largest number of central cells are "driven" by both eyes.

Dr. Arden described the visually evoked responses in human amblyopes. He had used as the visual stimulation a chess-board pattern, the white and black squares being alternately and intermittently illuminated. He showed that, in hysterical blindness, normal sinusoidal curves occurred, whereas in organic retinal lesions the response was abnormal. He also showed that, when the electrical responses from the two visual cortices were "in phase", binocular function was likely to be either normal or of a subnormal but useful type (e.g. anomalous retinal correspondence with anomalous fusion), whereas, when they were 'out of phase", binocularity was absent.

Dr. Daroff clarified the mechanisms involved in ocular movements and observed that, in acquired brain disease causing defects of pursuit movements, the patient himself does not complain of the disability. Nevertheless observation of such an anomaly may be most useful as an objective and localizing physical sign.

Miss Mein stated that visuscopic examination was necessary in all amblyopes in order to record fixation, bearing in mind that eccentricity of fixation often occurs with visual 
acuity as good as 6/12. She also stressed the importance of recording visual acuity for near as well as distance. She called for simplicity in the investigation of binocular problems, underlining the value of simple tests for stereoscopic acuity, and the use of prisms in estimating binocular function. She showed the value of demonstrating the extent of the field of binocular fixation using a perimeter in cases of paralytic squint in adults.

In the case of children suffering from strabismus associated with multiple developmental handicaps, Mr. Harcourt stressed the psychological advantage to both child and parents of improving the ocular deviation by surgery and so relieving the child of one of his disabilities.

Dr. Marshall Parks, with characteristic clarity, described the indications for bifocal glasses in the case of children with accommodational esotropia. He explained how such glasses should be dispensed and the role of the ophthalmologist in making sure that the child knows how to manage them. He advocated a "full" correction of the hypermetropia as determined under cycloplegia by retinoscopy with the subtraction of only I $\mathrm{D}$ sph. to allow for the fact that the examination is carried out at a distance of I metre. The reading addition should usually be $+2 \cdot 5 \mathrm{D}$ sph. For miotic therapy he found that di-isofluoropropyl 0.025 per cent. is the best drug if used first thing in the morning. He did not however mention the role that surgery can sometimes play in certain cases of accommodational esotropia as mentioned by Mr. Whitwell. For instance, although surgery (e.g. medial rectus recession) may be contraindicated in a patient who shows excessive convergence for near but orthophoria for distance, it may be most helpful for a patient who shows excessive convergence for near with considerable esophoria for distance. For, by relieving the esophoria for distance, the esotropia for near may be greatly reduced and indeed may possibly be controlled by a smaller additional spherical correction or even with none.

In like manner, although convergence insufficiency in itself cannot be cured by resection of the medial rectus muscles (any attempt to do so will give rise to exotropia for distance), yet convergence insufficiency existing in conjunction with a large degree of exophoria for distance may be considerably improved by lateral rectus recession.

Dr. Parks also mentioned the value of the relatively simple Jensen procedure combined with recession of the direct antagonist in the treatment of sixth nerve palsy.

However, Mr. Fells preferred the transplantation of the entire superior and inferior rectus insertions into the sclera immediately above and below the insertion of the affected lateral rectus, preceded by a full recession of the direct antagonist. For the surgical correction of excyclotropia, especially that due to bilateral superior oblique palsy (caused by head injury), he followed the technique of Harada and Ito of Japan, in advancing the anterior half of the superior oblique.

Mr. Graham reported that his epidemiological studies showed that heredo-familial factors were of primary importance in the causation of strabismus. There was no evidence that an adverse environment or sub-standard living conditions had any causative influence. In the sample of squinters he had studied, eso-deviation was three times commoner than exo-deviation.

Professor Bagolini, in discussing the significance and management of anomalous retinal correspondence, pointed out that the condition was essentially an adaptation to the presence of an ocular deviation whereby a newly acquired orientation was developed. Different methods of testing give different results because, in order to make the patient aware of the image of the deviating eye, an artefact has to be introduced. The Bagolini striated glasses, which are now regarded as of the greatest importance in the evaluation of cases of strabismus, involve a negligible artefact and are therefore especially useful. 
In patients with a small angle of squint with anomalous retinal correspondence binocular co-operation is good and the areas of anomalous retinal correspondence constitute a "pseudo-Panum's area" which is much larger than the normal Panum's area.

Dr. Lang clarified the condition of microtropia, saying that the angle of deviation in the condition as he understood it was always less than $5^{\circ}$ and that it was accompanied by harmonious anomalous retinal correspondence.

It was refreshing to find that, although there are some differences in detail and emphasis, the condition described as "microtropia" by Lang, that described as "monofixational phoria" by Marshall Parks, and that referred to as "microstrabismus" by von Noorden are in certain basic essentials very similar, the main point being that there is a type of esotropia of a small angle in which anomalous retinal correspondence exists with useful anomalous binocular function (including a good range of fusion and stereopsis), for which surgical treatment is usually not indicated and in which the anomalous binocular function should be left undisturbed.

Mr. Wybar gave a clear account of the AC/A ratio and described the various ways in which this can be accurately estimated. He also clearly defined the phenomenon of "fixation disparity" as described by Martens and Ogle (1959).

Dr. Durston gave a well-illustrated histological demonstration of the changes that occur in ocular muscle fibres after experimental damage to their nerve supply.

Dr. Huber, in his electrophysiological demonstration of cases of the retraction syndrome, suggested a possible embryological explanation of the "co-contraction mechanism". He thinks that it is likely to be caused by a branch of the third nerve (which in the $7 \mathrm{~mm}$. embryo is the only cranial nerve which has reached the muscles it supplies) coming into contact with that part of the ocular muscle mass which is destined to become the lateral rectus. (The fourth and sixth nerves do not reach their respective muscles until later in the stage of development.) Thus the lateral rectus may have two nerve supplies, one from the third nerve and one from the sixth, and it may therefore "behave like a lateral rectus" and also "behave like a medial rectus". In this connection we have the fact that Hoyt and Nachtigäller (1965a, b) reported that chance observations in the anatomical dissecting room disclosed an anomalous branch of the third nerve supplying the lateral rectus.

As regards nomenclature, Mr. Nutt pointed out that for historical accuracy the names of "Türck and Stilling" should be associated w th the retraction syndrome rather than, or in addition to, that of Duane.

Dr. Gobin, in discussing the management of the retraction syndrome, referred to the operation of vertical muscle transplantation combined with medial rectus recession to improve abduction in cases in which there is an obvious limitation of lateral movement. He had done this operation many times and had encountered no signs of impairment of circulation in the anterior segment of the eyeball. He stressed the importance of gentle manipulation and the maintenance of a bloodless operating field. He illustrated his paper with excellent slides.

Dr. Dunlap told us how to try to avoid the formation of scar tissue and adhesions in muscle surgery, how to remove them when they do occur, and how to prevent their recurrence. To help to avoid bleeding he advocated the use of Epinephrin I : I, ooo drops preoperatively, and to prevent adhesions he described the use of "Supramid extra" in the form of a sheet, sleeve, or cap placed between muscles and eyeball or between conjunctiva and underlying tissue. He also referred to the use of butylcyano-acrylate which forms a plastic film by polymerization when painted onto a surface.

He particularly stressed the importance of meticulously careful surgery. 
The whole symposium has been of great value not only for the high standard of its communications and for the informality of the subsequent discussions, but also for the fact that plenty of time was available between sessions for talking to old friends and for making new ones in the congenial atmosphere of one of the most dignified colleges in Cambridge. 